\title{
Design of the Magnetic Field Sensing System for Downlink Signal Reception and Interference Cancelling for Through-the-Earth Communication
}

\author{
Peng Zhao, Yu-zhong Jiang*, Shu-xia Zhang, and Wen-wei Ying \\ College of Electronic Engineering, Naval University of Engineering, Wuhan 430033, China
}

(Received 7 July 2016, Received in final form 15 August 2016, Accepted 16 August 2016)

\begin{abstract}
A magnetic field sensing system with a single primary sensor and multiple reference sensors deployed locally and orthogonally, was proposed for downlink signal reception and interference cancelling for Through-theEarth Communication (TEC). This paper mathematically analyzes a design optimization process for a search coil magnetometer (SCM), and applies that process to minimize the bandwidth of the primary SCM for TEC signal reception and the volume of reference SCMs for multiple distributions. The primary SCM achieves a 3dB bandwidth of $7 \mathrm{~Hz}$, a sensitivity threshold of $120 \mathrm{fT} / \sqrt{\mathrm{Hz}}$, and a volume of $2.32 \times 10^{-4} \mathrm{~m}^{3}$. The entire sensing system volume is as small as $10^{-2} \mathrm{~m}^{3}$. Experiments with interference from industrial frequency harmonics demonstrated an average of $36 \mathrm{~dB}$ and $18 \mathrm{~dB}$ improvements in signal-to-interference ratio and signal-tointerference plus noise ratio, respectively, using multichannel recursive-least-squares algorithm. Thus, the proposed sensing system can reduce the interference effectively and allows reliable downlink signal reception.
\end{abstract}

Keywords : magnetic field sensing, Through-the-Earth Communication (TEC), downlink signal reception, interference cancelling

\section{Introduction}

Through-the-Earth Communication (TEC) benefits from the superior penetration ability of low-frequency electromagnetic signals and is considered one of the most effective approaches for emergency communications in both underground and underwater terrains. It is operated in the frequency range of $10 \mathrm{~Hz}$ to $10 \mathrm{kHz}$, in which atmospheric noise and man-made electromagnetic interference (EMI) are severe. Further, the radiation efficiency of the transmitting antennas is very low (lower for portable antennas), and the received signal level tends to be several orders lower than the EMI. Thus, the ability to communicate is mainly dependent on the signal-to-noise ratio (SNR) at the receiving terminals [1].

In previous research on trapped-miner communication and location systems based on TEC, the receiving system was mainly built using local three-axial primary receiving (for the uplink communication signal) and remote reference (for the correlated interference) magnetic-field antennas. Several signal processing techniques have been

(C)The Korean Magnetics Society. All rights reserved.

*Corresponding author: Tel: +86-13657291589

Fax: +86-02783635069, e-mail: jiangyuzhong@tsinghua.org.cn used to reduce interference, such as an adaptive clipper combined with direct matrix inversion achieving average $4.1 \mathrm{~dB}$ improvement [2] for interference at extremely-low frequency (ELF, $3 \mathrm{~Hz}-30 \mathrm{~Hz}$ ), and adaptive noise cancelling combined with maximum-likelihood detection, nonlinear processing, and decision-aided feedback achieving 10-to-24 dB improvement [3] for interference at verylow frequency (VLF, $3 \mathrm{kHz}-30 \mathrm{kHz}$ ). To date, the trappedminer communication and location systems continue to encounter many challenges, of which the key research focuses on digital signal processing and interference cancelling techniques that could be effective at these low frequencies [1].

However, there is little research published on TEC downlink signal reception, especially regarding EMI cancelling. This may be partly because EMI will disappear at the downlink signal receiving sites such as mines, when emergencies. However, in cases such as underwater communications with Unmanned Underwater Vehicles (UUVs) from the surface or the corresponding point-to-point underwater wireless communications, the received signal is coupled with significant EMI generated by the UUV itself $[4,5]$, which makes EMI cancelling indispensable at such downlink sites.

In general, terrains underground or underwater, such as 
the terrains in deep mines and seabed, are complicated and the space is confined. Therefore, it is difficult, inconvenient, and costly to place noise-reference sensors remotely when communicating. Further, sensors such as loop antennas [1, 6], air coils, and electrodes [7, 8] are unsuitable for placement in underground or underwater terrains, especially during emergencies [9]. This is because air coils or loop antennas need to be large (such as these loop diameters of $3.8,12.5,26$, and $90 \mathrm{~m}$ [1]) to obtain high sensitivity required for receiving weak magneticfield signals, and the receiving terminals of the electrodes need to be separated over a large distance. All these receiving sensors occupy a very large area or volume that is usually unavailable in underground or underwater terrains. Thus, the receiving systems mentioned above are infeasible for TEC downlink signal reception and EMI cancelling.

Adopting a ferromagnetic core effectively increases air coil sensitivity without enlarging the coil size [10]. Currently, most ferromagnetic-cored coil designs focus on their wideband performance [11-13]. However, TEC requires very narrow bandwidth, i.e., a few hundred Hertz in VLF and a few Hertz in super-low frequency (SLF, 30 $\mathrm{Hz}-300 \mathrm{~Hz}$ ). Thus, one approach to design the SCM more suitable for TEC, especially for SLF and lower frequencies, is paralleling the matching capacitor and tuning the resonant frequency to the TEC carrier frequency [9]. Besides, the coils need to be small in size so as to meet the demands of easy placement and portability [14].

In this study, a novel magnetic field sensing system with a single primary receiving and multiple-reference ferromagnetic-cored SCMs deployed locally and orthogonally, was proposed and designed for TEC downlink signal reception and interference cancelling. The primary receiving SCM was optimized to minimize the bandwidth, which was subjected to a given sensitivity and volume, while the reference SCMs were optimized to minimize the volume, which was subjected to the given sensitivity and bandwidth. The sensing system was built using these SCMs and then tested. With the analog-to-digital converter (AD7609) and the DSP-FPGA platform to process the output of the SCMs, experiments with the EMI from industrial frequency harmonics (IFHs) were conducted. The experiments demonstrated an average of $36 \mathrm{~dB}$ and $18 \mathrm{~dB}$ improvements in the signal-to-interference ratio (SIR) and the signal-to-interference plus noise ratio (SINR), respectively, using the multichannel recursive-least-squares (RLS) algorithm. Compared with Ref. [2] and [3], which employed remote noise-reference sensors, the proposed sensing system achieved a better performance for EMI cancelling, which features a compact structure and a volume of only $10^{-2} \mathrm{~m}^{3}$. Thus, the proposed system is effective for downlink signal reception and EMI cancelling for TEC.

\section{Analysis of the EMI and Design of the Sensing System}

\subsection{Analysis of the EMI and cancelling strategies}

TEC is mainly influenced by atmospheric noise and man-made EMI. Atmospheric noise is mostly produced by lightning discharges and it propagates worldwide. The waveform received by typical receivers features strong and clustered impulses [15] with a Gaussian background noise, and the power spectrum is flat at low frequencies. It is non-stationary and varies based on seasons, days, geographic locations, and geology [16]. The man-made EMI mainly consists of IFHs, which are generated by power lines and vary by days and geographic locations. The power spectrum comprises spikes at specific frequencies, which are positive integer multiples of the fundamental frequency [15]. Therefore, the total EMI appearing in the TEC downlink sites is directional and random in the spatial domain, impulsive and non-stationary in the time domain, and, in the frequency domain, flat for atmospheric noise while uneven for the EMI produced by IFHs.

It is usually much more difficult to perform EMI cancelling with conventional fixed-parameter filters owing to inadequate priori knowledge, despite several statistical models [15-18] of the EMI. Adaptive noise cancelling (ANC), which adjusts its own weight parameters adaptively and thus, requires little or even no priori knowledge [19], seems appropriate for TEC EMI cancelling at downlink sites.

Because more than one type of EMI exists in the TEC downlink signal and the output of the noise-reference SCMs can be linearly independent, the multiple-reference

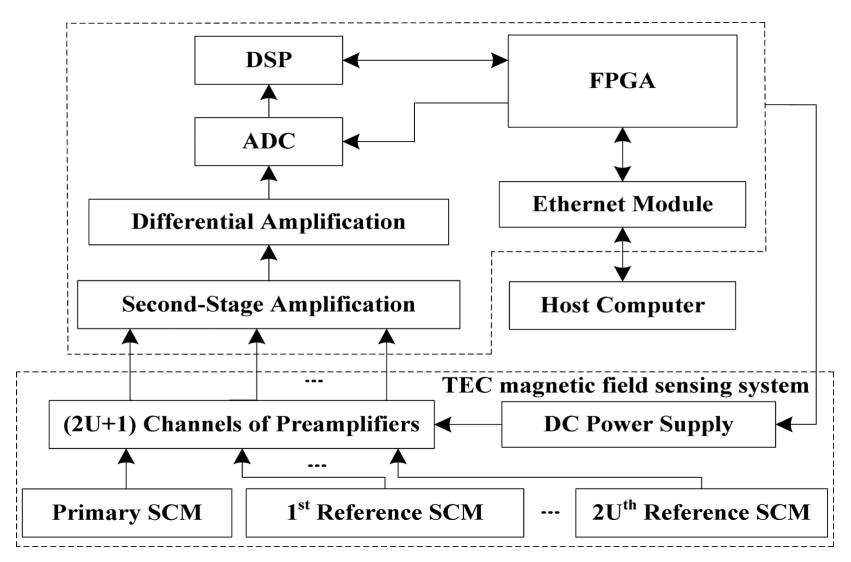

Fig. 1. Block diagram of TEC receiving system. 
noise cancelling system [19] is adopted in this study, and it is shown in the block diagram of the receiving system (Fig. 1).

As shown in Fig. 1, the TEC magnetic field sensing system consists of one primary and $2 U(U \geq 1)$ reference $\left(1^{\text {st }}-2 U^{\text {th }}\right)$ SCMs, and it is the focus of this research.

\subsection{Structural design of the magnetic field sensing} system

According to the principles of ANC, signal components leaking into the reference inputs lead to the signal distortion and the decline of the SIR gain at the canceller output [19]. Therefore, the reference SCMs should be deployed such that the induced TEC signal components are minimized. This may be realized by separation between the primary receiving and reference sensors above the surface. However, the separation seems infeasible in underground or underwater terrains due to space limitations. Another effective method involves placing the reference SCMs locally and orthogonally with the primary receiving SCM. When the transmitting antenna and the primary receiving SCM are oriented in the same direction, the TEC signal transmission is optimized [1]. Thus, the signal components can be maximized in the primary receiving SCM while they are minimized in the reference SCMs.

We proposed a structure of a TEC magnetic field sensing system for downlink signal reception and EMI cancelling, and this structure is shown in Fig. 2. With the single primary receiving and multiple reference SCMs deployed locally and orthogonally, we can conserve space and make the whole system compact by "locally", and help minimize the signal components leaking into the reference inputs through orthogonality when the transmitting antenna and primary receiving SCM are oriented in the same direction. In addition, the adoption of multi-

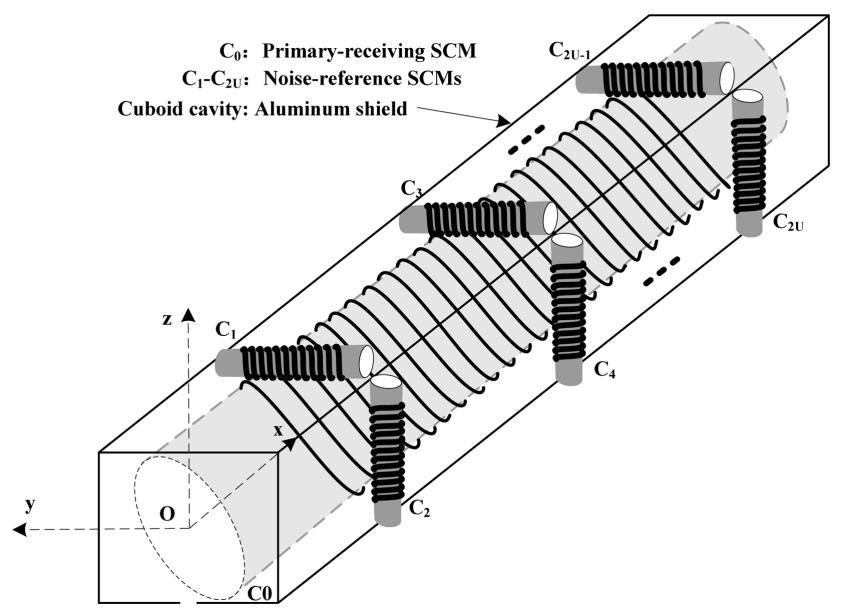

Fig. 2. Structure of the magnetic field sensing system.

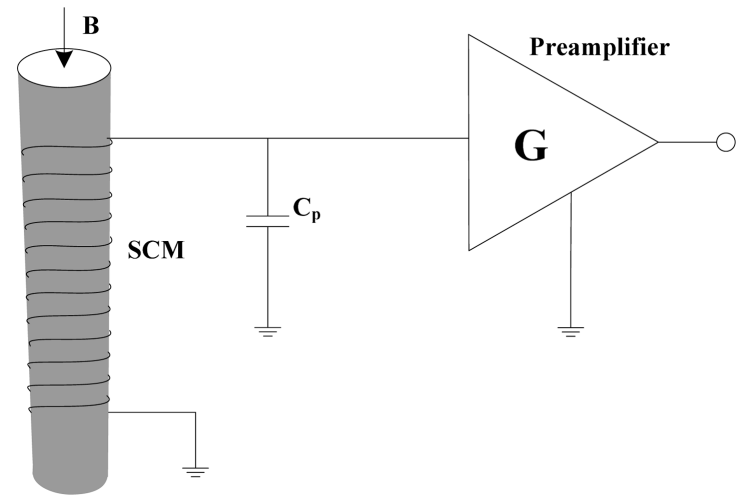

Fig. 3. SCM combined with preamplifier.

ple reference SCMs is advantageous because more than one type of interference may be present.

As shown in Fig. 2, the primary receiving SCM is parallel to the $\mathrm{x}$-axis and wrapped with an aluminum shield in the shape of a cuboid cavity for high isolation from the electric field, whereas the reference SCMs $\mathrm{c}_{1}$ to $\mathrm{c}_{2 \mathrm{U}-1}$ and $\mathrm{c}_{2}$ to $\mathrm{c}_{2 \mathrm{U}}$ are placed parallel to the $\mathrm{y}$-axis and $\mathrm{z}$ axis, respectively, and affixed to the outer surface of the cuboid cavity. There is a narrow seam at the bottom of the outer surface of the cuboid cavity to avoid the galvanic surface current. Each axis of the vectoral sensing system is designed as a coil of copper wire with a ferromagnetic core inside [20], which is followed by the preamplifier (Fig. 3).

In Fig. 3, $C_{p}$ represents the matching capacitor, which is used for tuning the resonant frequency. The preamplifier is supposed to be high-gain and low-noise to relieve the impact of the rear-stage circuit on the total noise figure.

\section{Optimization of the SCMs}

\subsection{Analysis of the SCM response and its noise}

The SCM is described by the series of the resistor $r_{s}$, the inductor $L_{s}$ and the capacitor $C_{s}$. The equivalent

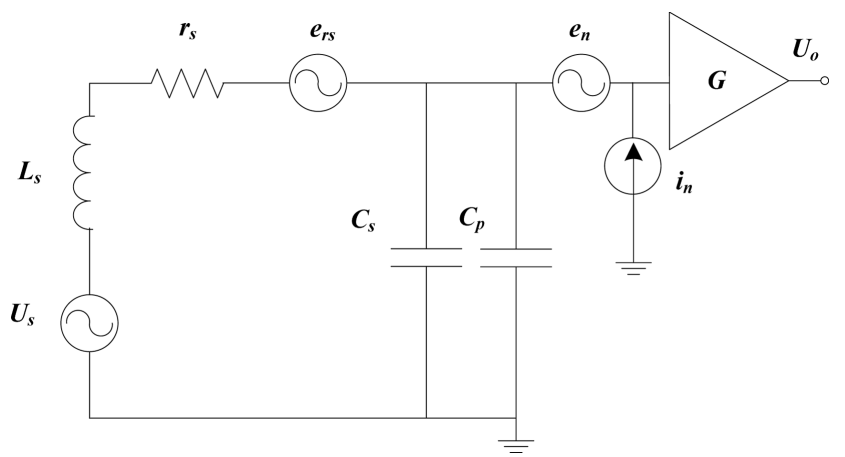

Fig. 4. Equivalent schematic of SCM and the noise sources. 
schematic of Fig. 3 is shown in Fig. 4 with the noise sources presented.

In Fig. $4, e_{r s}$ represents the thermal noise of $r_{s}$, and $e_{n}$ and $i_{n}$ are the equivalent input voltage noise and current noise of the preamplifier, respectively. The gain of the preamplifier is $G$, and its output is $U_{o}$. The induction voltage of the SCM is represented by $U_{s}$ and is calculated as (1) in the frequency domain according to Faraday's law.

$$
U_{s}(f)=-j 2 \pi f \mu_{a} N A B,
$$

where $B$ is the magnetic flux density along the axis of the core, $\mu_{a}$ is the apparent permeability, $f$ is the frequency, $N$ is the number of turns, and $A$ is the cross-sectional area of the core.

$U_{s}(f)$ passes through the RLC second-order network and the preamplifier to $U_{o}(f)$, and thus the frequency response of the SCM can be deduced as

$$
\frac{U_{o}(f)}{B}=\frac{u_{a} N A G}{-r_{s} C+j\left(\frac{1}{2 \pi f}-2 \pi f L_{s} C\right)},
$$

where $C=C_{p}+C_{s} . f_{0}$ represents the resonant frequency, and

$$
f_{0}=\frac{1}{2 \pi \sqrt{L_{s} C}} .
$$

The $3 \mathrm{~dB}$ bandwidth $f_{B}$ of the SCM can then be calculated by

$$
\frac{\left|U_{o}\left(f_{0}\right)\right|^{2}}{\left|U_{o}\left(f_{0}-\Delta f\right)\right|^{2}}=2,
$$

where $\Delta f=f_{B} / 2$. By combining (2) and (4), we can express the bandwidth as

$$
2 \pi\left(\Delta f+\frac{f_{0} \Delta f}{f_{0}-\Delta f}\right) L_{s}=r_{s} .
$$

It is important to note that when $f_{0}$ is more than one order larger than $\Delta f$, as in the case of VLF, we can obtain $\Delta f / f_{0} \approx 0$ and thus the bandwidth $f_{B} \approx r_{s} / 2 \pi L_{s}$. However, in the cases of SLF and ELF, such a condition could not always be satisfied, and the bandwidth would be deduced directly from (5) and obtained as

$$
f_{B}=2 f_{0}+\left(\tau-\sqrt{4 f_{0}^{2}+\tau^{2}}\right),
$$

where $\tau=r_{s} / 2 \pi L_{s}$. Note that there should be two values of $f_{B}$ in (6), but the bandwidth should not be larger than $2 f_{0}$; therefore, $\tau+\sqrt{4 f_{0}^{2}+\tau^{2}}$ has not been mentioned.

Finally, the equivalent magnetic field noise of the SCM is analyzed. From Fig. 4, the contribution of $e_{n}$ to the equivalent input voltage noise of the SCM is

$$
e_{e n}=\left[1+j 2 \pi f C\left(r_{s}+j 2 \pi f L_{s}\right)\right] e_{n} .
$$

The contribution of $i_{n}$ to the equivalent input voltage noise of the SCM is

$$
e_{i n}=\left(r_{s}+j 2 \pi f L_{s}\right) i_{n} .
$$

Thus, the spectral density of the total noise referred to the SCM input can be calculated as

$$
\left|e_{\text {total }}\right|=\sqrt{\left|e_{e n}\right|^{2}+\left|e_{i n}\right|^{2}+\left|e_{r s}\right|^{2}},
$$

where, $e_{r s}=\sqrt{ } 4 k \operatorname{Tr}_{s}, T$ is the temperature in Kelvin and $k$ is the Boltzmann constant. $\left|e_{n}\right|$ and $\left|i_{n}\right|$ denote the effective values of $e_{n}$ and $i_{n}$ respectively.

When we set (9) to be equal to (1), the sensitivity threshold [12] (also called the noise equivalent magnetic induction [20]) $B_{s t}$ can be obtained as

$$
B_{s t}(f)=\frac{\left|e_{\text {total }}\right|}{2 \pi f \mu_{a} N A} .
$$

The sensitivity threshold is an equivalent magnetic induction that determines the lowest detectable magnetic field beyond the background noise for the SCM. The lower the sensitivity threshold, the higher is the sensitivity.

According to the structure and dimensions of the SCM in Ref. [12], we can calculate the DC resistance, inductance [10], and volume of the SCM as follows:

$$
\begin{aligned}
r_{s} & =\rho \frac{2\left(b+h+2 \frac{N \beta^{2} d_{w}^{2}}{a \gamma}\right) N}{\pi d_{w}^{2} / 4} \\
& =\frac{8 \rho\left(v a \gamma+2 N \beta^{2} d_{w}^{2}\right) N}{\pi a \gamma d_{w}^{2}} \\
L_{s} & =\frac{\mu_{0} \mu_{a} \gamma^{-3 / 5} A N^{2}}{a} \\
V & =\gamma a\left(\chi^{2}+v \chi+A\right) \\
\chi & =\beta\left(2 \frac{N \beta d_{w}}{\gamma a}-1\right) d_{w}{ }^{2}
\end{aligned}
$$

where the core is assumed to be in the shape of a cuboid with length $a$, width $b$ and height $h$; the cross-sectional area $A$ of the core is $b \times h ; v=b+h$ in (11) and (13) for simplification; $V$ denotes the volume of the SCM; $d_{w}$ is the diameter of the copper wire without insulation; $\gamma$ represents the ratio of the winding length to the core length; $\rho$ is the resistivity of the copper wire; $\beta$ is the ratio of the total wire diameter with insulation to the copper 
wire diameter without insulation; and $\mu_{0}$ is the vacuum permeability.

\subsection{Solution to the SCM optimization problem}

In this study, firstly, the carrier frequency was set to 85 $\mathrm{Hz}$ for TEC using SLF, and the resonant frequency of the primary receiving $\mathrm{SCM}$ was tuned to this carrier frequency [9] by $C_{p}$. Thus, on one hand, the primary-receiving SCM can be designed for the desired narrow bandwidth. On the other hand, the interference beyond the bandwidth can be fully reduced and the maximal SNR at the preamplifier input can be obtained. Therefore, the bandwidth of the primary receiving SCM should be minimized to reduce the EMI effectively. The resonant frequency of the reference SCMs was tuned to $95 \mathrm{~Hz}$, and the $3 \mathrm{~dB}$ bandwidth was designed to be $14 \mathrm{~Hz}$ to acquire external EMI.

Secondly, theoretical predictions indicate that a sensitivity threshold of $100 \mathrm{fT} / \sqrt{\mathrm{Hz}}$ at $85 \mathrm{~Hz}$ for the primary receiving SCM should be sufficient for laboratory tests. In addition, the sensitivity threshold of the reference SCMs should be at least 10 times larger than the primary receiving SCM such that the TEC signal is not detected by the reference SCMs at the highest transmitting power in laboratory tests.

Finally, the volume of both the primary receiving and reference SCMs should be confined to conserve space. The volume of the primary receiving SCM should not be larger than $2.4 \times 10^{-4} \mathrm{~m}^{3}$ according to the volume of the cuboid cavity (Fig. 2), and the volume of the reference SCMs should be minimized to support multiple distributions.

To meet all of the above requirements, the SCM should be optimized. In this study, we assume that the size and relative permeability of the core, the ratio of the winding length to the core length, the ratio of the total wire diameter with insulation to the copper wire diameter without insulation, and the noise of the preamplifier are given before optimization. In such a case, the only independent variables are the diameter of the copper wire $d_{w}$ and the number of turns $N$.

Thus, the optimization problem becomes the optimization of $d_{w}$ and $N$ that minimizes the bandwidth for the primary receiving SCM, which is subjected to a given volume and sensitivity threshold as

$$
\left\{\begin{array}{l}
\min f_{B}\left(N, d_{w}\right) \\
\text { subjected to } B_{s t}\left(N, d_{w}\right)=10^{-13} \text { and, } \\
V\left(N, d_{w}\right)=2.4 \times 10^{-4}
\end{array}\right.
$$

For reference SCMs, the optimization goal is to minimize the volume, which is subjected to a given bandwidth and sensitivity threshold as $\left\{\begin{array}{l}\min V\left(N, d_{w}\right) \\ \text { subjected to } B_{s t}\left(N, d_{w}\right)=10^{-12} \text { and, } \\ f_{B}\left(N, d_{w}\right)=14\end{array}\right.$

Both $r_{s}$ and $L_{s}$ in (6), (7), and (8) are dependent variables of $d_{w}$ and $N$ according to (11) and (12). This is the same as the case for $C_{p}$ since $C_{p}=\left(2 \pi f_{0}\right)^{-2} L_{s}^{-1}$, where $C_{s}$ is negligible for small $f_{0}$ in (3).

In this work, the core material is stalloy and the typical relative permeability is 5000 for very weak magnetic fields at low frequencies. The dimensions of the core are $35 \times 33 \times 160 \mathrm{~mm}$ and $9.6 \times 12.6 \times 57 \mathrm{~mm}$ for the pri-

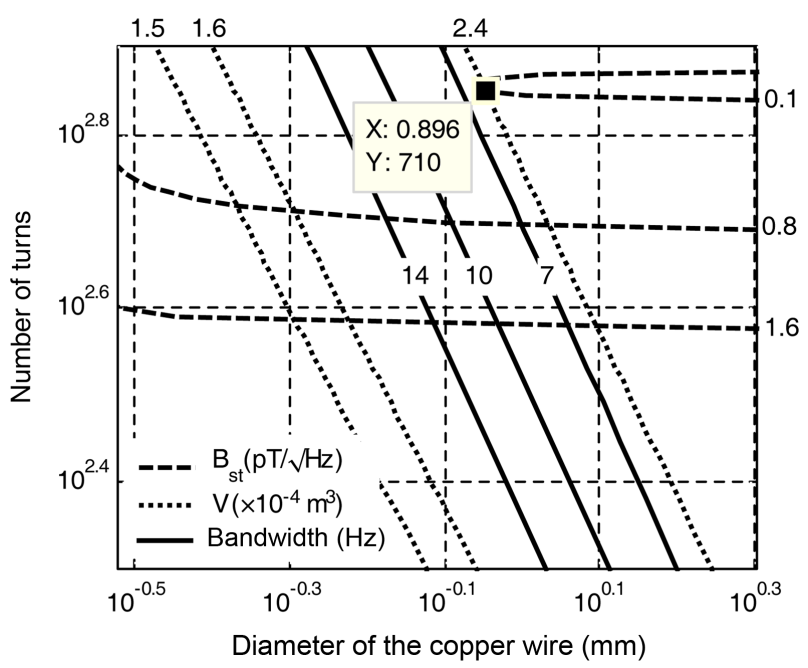

Fig. 5. (Color online) Contour maps of the sensitivity threshold, bandwidth and volume of the primary receiving $\mathrm{SCM}$ at $85 \mathrm{~Hz}, \mathrm{~T}=293 \mathrm{~K}$.

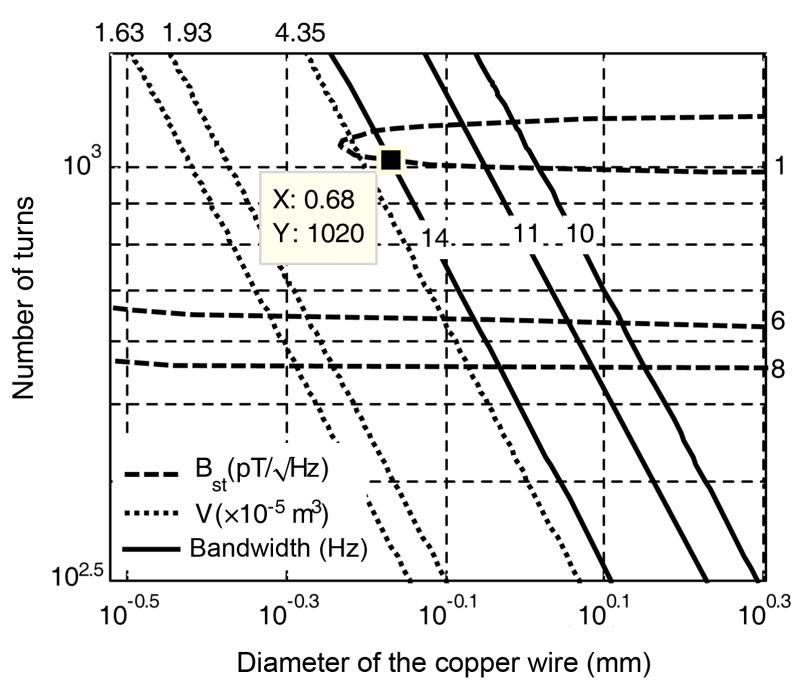

Fig. 6. (Color online) Contour maps of the sensitivity threshold, bandwidth and volume of the reference SCM at 95 $\mathrm{Hz}, \mathrm{T}=293 \mathrm{~K}$. 
mary receiving and reference SCMs, respectively. The ratio of the total wire diameter with insulation to the copper wire diameter without insulation is 1:0.9 for all SCMs. The preamplifier circuit is mainly composed of two bipolar transistors $2 \mathrm{~N} 930$, and the measured $e_{n}$ and $i_{n}$ are $6.82 \mathrm{nV} / \sqrt{\mathrm{Hz}}$ and $4.76 \mathrm{pA} / \sqrt{\mathrm{Hz}}$, respectively, at $85 \mathrm{~Hz}$.

In general, the Lagrange multipliers method could be used for solving (14) and (15), and the Newton algorithm could be used for the optimizing process [21]. In this study, contour maps $[9,13]$ are adopted, and the results are shown in Figs. 5 and 6 for the primary receiving and reference SCM, respectively.

We can easily find the optimum diameter of the copper wire and number of turns of the primary receiving SCM from Fig. 5, and they are $d_{\text {wopt }}=0.896 \mathrm{~mm}$ and $N_{\text {opt }}=710$ turns. Similarly, for the reference SCMs, Fig. 6 shows $d_{\text {wopt }}=0.68 \mathrm{~mm}$ and $N_{\text {opt }}=1020$ turns. Note that the crosssectional shape of the core is equivalent to a circular cross section $[11,13]$ for the calculation of $L_{s}$. The core has been cut into long, thin strips along the $\mathrm{x}$-axis in order to decrease the eddy current [13].

\section{Experiments and Discussion}

To verify the optimization process, we built experimental models of the SCMs whose parameters were chosen as close as possible to the optimum parameters. Then, these SCMs were employed to build the sensing system as shown in Fig. 2. The frequency response and sensitivity threshold of the SCMs were tested, and the results are summarized in this section. In addition, the test results for the performance of signal reception and EMI cancelling of the sensing system are presented.

\subsection{Test of the frequency response and sensitivity} threshold

A square Helmholtz Coil [22-24] calibration system driven by the spectrum analyzer SR785 was used to test the frequency response of the SCMs. Each coil is $2 \mathrm{~m}$ of side length, and the distance between the two coils is $1 \mathrm{~m}$. The total field inhomogeneity is less than $2 \%$ within an area of $30 \mathrm{~cm}$ of the axial length and $40 \mathrm{~cm}$ of radial length within the frequency range of 10-500 Hz. The Helmholtz Coil was built in a shielding room so that the man-made EMI would be reduced to a negligible level. The experimental results are shown in Fig. 7 for the primary receiving SCM and Fig. 8 for the reference SCM with the simulation included.

Figures 7 and 8 show that there exist some differences between the experimental and predicted plots. On one hand, the measured inductance and matching capacitor

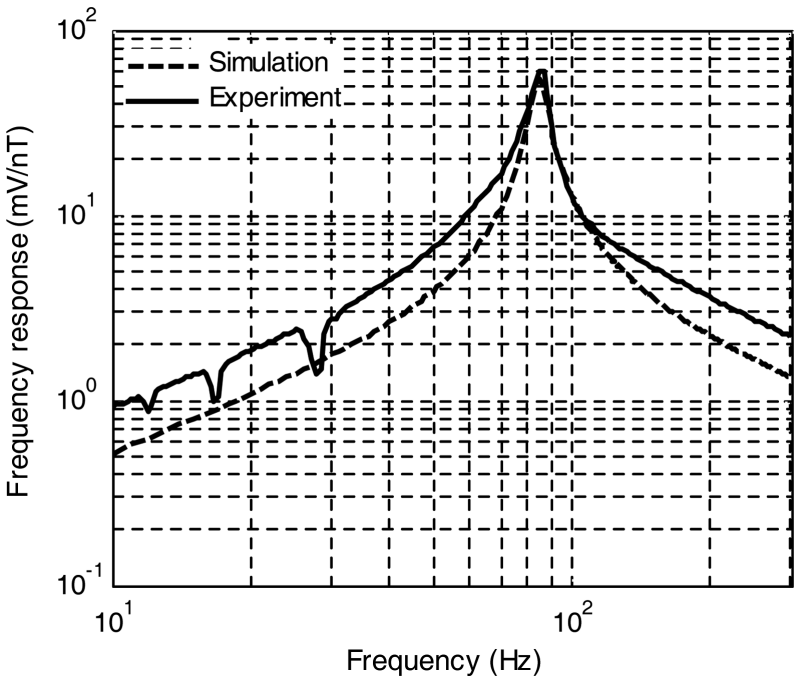

Fig. 7. Frequency response of the primary receiving SCM.

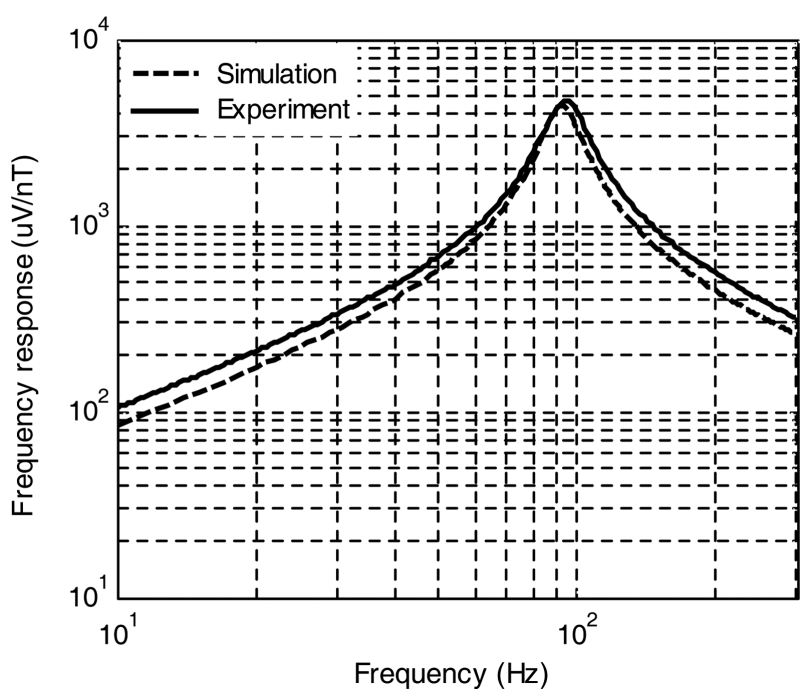

Fig. 8. Frequency response of the reference SCM.

values do not exactly match the simulation, and therefore, the resonant frequency of the experimental models is slightly different from the theoretical models. On the other hand, since the DC resistance of the coil is small, the magnetic core losses cannot be neglected at low frequencies [11]. Thus the quality factor of the coil is decreased, and the bandwidth is broadened.

The sensitivity threshold was tested inside a cubical box [11], which is made of a multi-layer high-permeability permalloy material. The results are shown in Fig. 9 and 10 for the primary receiving and reference SCMs, respectively, along with the simulation results.

Figures 9 and 10 indicate that the shapes of the experimental results and the simulation agree within some slight error. The error is minimum within the $3 \mathrm{~dB}$ bandwidth 
and increases for lower and higher frequencies. The discrepancies are believed to be partly attributed to the preamplifier noise. The voltage noise and current noise of

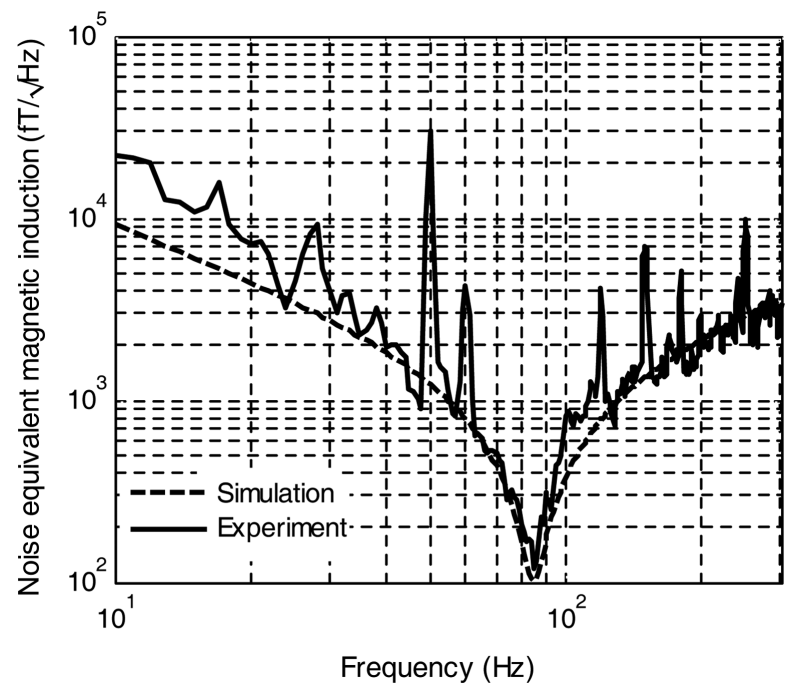

Fig. 9. Sensitivity threshold of the primary receiving SCM.

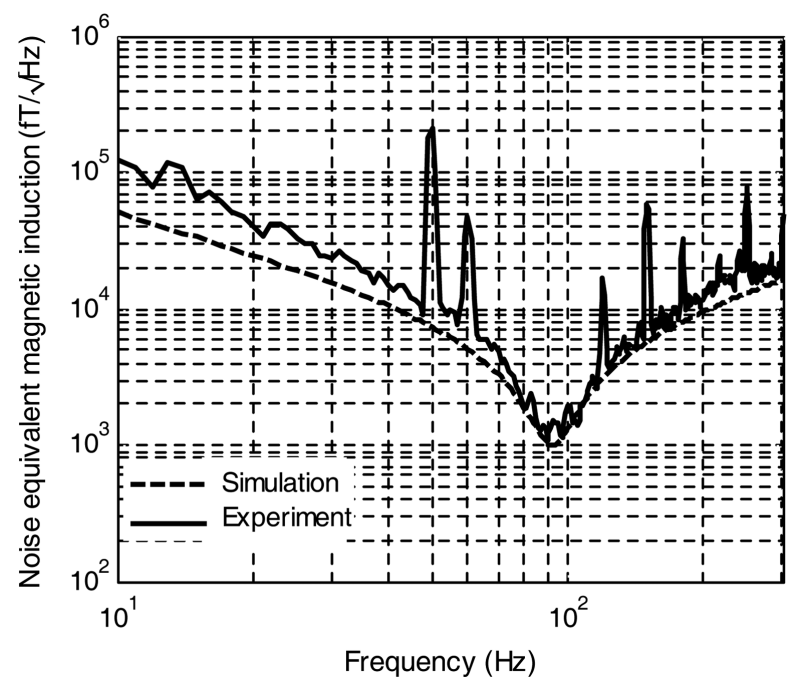

Fig. 10. Sensitivity threshold of the reference SCM.

Table 1. Parameters of the experimental models of the SCMs.

\begin{tabular}{lcc}
\hline \hline \multicolumn{1}{c}{ Parameters } & Primary SCM & Reference SCM \\
\hline$d_{w}(\mathrm{~mm})$ & 0.85 & 0.60 \\
$N($ turns $)$ & 720 & 1100 \\
$C_{p}(\mathrm{uF})$ & 45 & 57 \\
$f_{0}(\mathrm{~Hz})$ & 85 & 95 \\
$r_{s}(\mathrm{Ohm})$ & 3.51 & 5.23 \\
$L_{S}(\mathrm{mH})$ & 79 & 52 \\
$V\left(\mathrm{~m}^{3}\right)$ & $2.32 \times 10^{-4}$ & $4.42 \times 10^{-5}$ \\
$f_{B}(\mathrm{~Hz})$ & 7 & 16 \\
$B_{s t}(\mathrm{fT} / \sqrt{ } \mathrm{Hz})^{\mathrm{a}}$ & 120 & 1435 \\
\hline
\end{tabular}

\footnotetext{
${ }^{\mathrm{a}}$ At resonant frequency
}

the preamplifier is dependent on frequencies and collector currents [25], while only a typical measured value at 85 $\mathrm{Hz}$ was used as a constant in the simulation throughout the frequency ranges for simplification. In addition, the $1 / f$ noise of the bipolar transistors would increase at lower frequencies [25], which could lead to a higher error in the frequencies lower than the resonant frequency. Spikes at multiples of $50 \mathrm{~Hz}$ and $60 \mathrm{~Hz}$ came from the magnetic interference of the environment. The parameters of the experimental SCMs are listed in Table 1.

\subsection{Test of the sensing system for signal reception and EMI cancelling}

The sensing system was tested with real EMI from IFHs in a laboratory environment. Six reference SCMs (for $U=3$ ) were adopted, and the external view of the sensing system is shown in Fig. 11. The entire volume of the sensing system is as small as $10^{-2} \mathrm{~m}^{3}$.

The $85-\mathrm{Hz}$ single tone signal was adopted as the TEC signal. The interference sources mainly consisted of household wiring in the laboratory, and their spatial distribu-

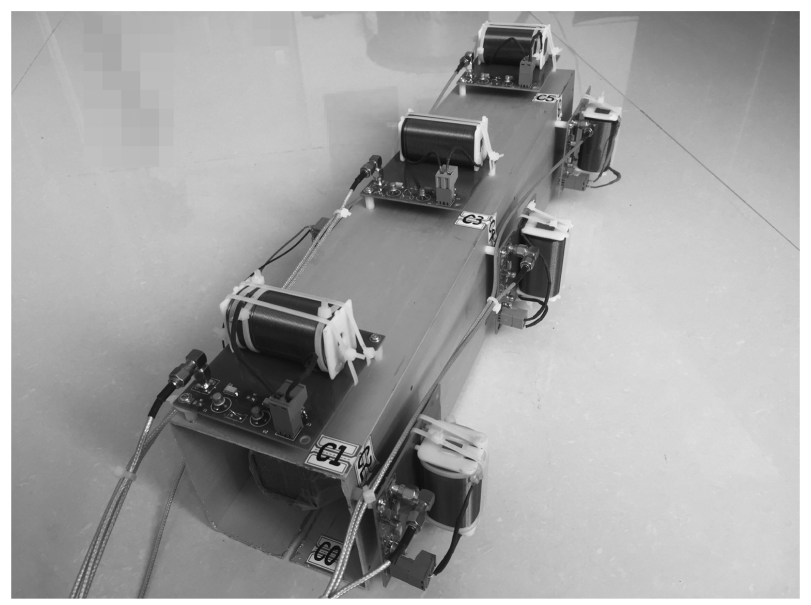

Fig. 11. External view of the sensing system.

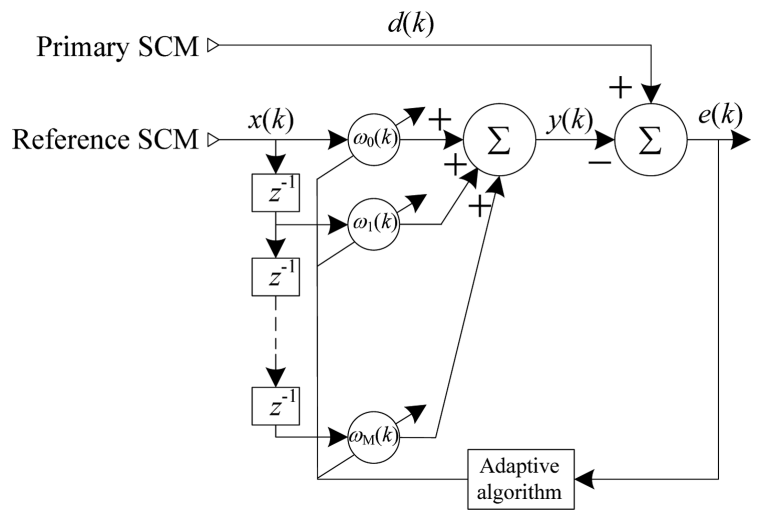

Fig. 12. Block diagram of ANC. 
tions were unknown. The sensing system was located at the center of the house and oriented randomly. An aircored square coil with $1 \mathrm{~m}$ of side length and 500 turns was used as the transmitting antenna. Both the transmitting and primary receiving coils were oriented in the same direction, with $5 \mathrm{~m}$ of distance between them. The Ethernet module in Fig. 1 was used for transceiving data between the lower computer and host computer for realtime processing. The $1.25 \mathrm{kHz}$ sample rate was adopted for the analog-to-digital conversion process. The output of the primary and reference SCMs, with 16384 samples from each, constituted a data set.

ANC techniques were used to process the data sets. The corresponding block diagram for a single-reference input is shown in Fig. 12, where $d(k)$ denotes the desired response at the discrete time of $k$ and consists of the primary SCM output, $x(k)$ denotes the reference input signal and consists of the reference SCM output, and $y(k)$ denotes the output of the adaptive filter. The output of the adaptive noise canceller is the a priori error signal $e(k)=$ $d(k)-y(k) . \omega(k)$ and $\boldsymbol{x}(k)$ represent the weight and input vector of the adaptive filter, respectively, and $\omega(k)=$ $\left[\omega_{0}(k), \omega_{1}(k), \ldots, \omega_{M}(k)\right]^{T}, \boldsymbol{x}(k)=[x(k), x(k-1), \ldots, x(k-M)]^{T}$, and $y(k)=\omega^{T}(k) \boldsymbol{x}(k)$, where $T$ denotes the transpose of the vector and $M$ is the order of the adaptive filter.

Two adaptive algorithms were adopted for EMI cancelling and they will be analyzed here. First, the singlereference algorithms are introduced, and then the multireference algorithms are derived through extensions of them.

One approach is the classic least mean squares (LMS) algorithm [19], which is widely used due to its low computational complexity. Consider the mean-square error (MSE) $E\left[e^{2}(k)\right][19]$

$$
\begin{aligned}
& M S E=\boldsymbol{\omega}^{T}(k) E\left[\boldsymbol{x}(k) \boldsymbol{x}^{T}(k)\right] \boldsymbol{\omega}(k)+ \\
& E\left[d^{2}(k)\right]-2 E\left[d(k) \boldsymbol{x}^{T}(k)\right] \boldsymbol{\omega}(k)
\end{aligned} .
$$

According to the steepest descent method, the weight vector is updated by the negative gradient of the MSE. Assuming that the MSE can be estimated by the square of the instantaneous error sample, the gradient would be calculated by differentiating $e^{2}(k)$ with respect to $\omega(k)$. Thus, the Widrow-Hoff LMS algorithm [19] is obtained as

$$
\boldsymbol{\omega}(k+1)=\boldsymbol{\omega}(k)+2 \mu e(k) \boldsymbol{x}(k),
$$

where $k \geq 0, \mu$ is the convergence factor and $0<\mu<1$ / $\lambda_{\max }$, and $\lambda_{\max }$ denotes the largest eigenvalue of the input correlation matrix. The initial values are $\omega(k)=[0,0, \ldots$, $0]^{T}$ and $\boldsymbol{x}(k)=[0,0, \ldots, 0]^{T}$.
The other approach is the recursive least squares (RLS) algorithm [26], which features fast convergence and excellent performance in a time-varying environment. Different from the minimization of the MSE shown in (16) in the LMS algorithm, the RLS algorithm aims at minimizing the cost function that is defined as

$$
c(k)=\varepsilon^{T}(k) \varepsilon(k),
$$

where $\varepsilon(k)=\left[\varepsilon(k), \lambda^{1 / 2} \varepsilon(k-1), \ldots, \lambda^{k / 2} \varepsilon(0)\right]^{T}$ is the exponentially weighted a posteriori error vector, $\varepsilon(l)=d(l)$ $\boldsymbol{\omega}^{T}(k) \boldsymbol{x}(l), l=k, k-1, \ldots, 0$, and $\lambda$ is the forgetting factor, $0<<\lambda \leq 1$.

According to Fig. 12, we can obtain $\varepsilon(k)=\boldsymbol{d}(k)-$ $\boldsymbol{X}(k) \boldsymbol{w}(k)$, where $\boldsymbol{d}(k)=\left[d(k), \lambda^{1 / 2} d(k-1), \ldots, \lambda^{k / 2} d(0)\right]^{T}$ and $X(k)=\left[x(k), \lambda^{1 / 2} \boldsymbol{x}(k-1), \ldots, \lambda^{k / 2} \boldsymbol{x}(0)\right]^{T}$. Therefore, the minimization of the cost function is equivalent to the minimization of the distance between vectors $\boldsymbol{d}(k)$ and $X(k) \omega(k)$. Thus, the optimal weight vector can be obtained in the least squares sense through the following equation.

$$
\boldsymbol{X}^{T}(k) X(k) \omega_{o p t}(k)=X^{T}(k) d(k) .
$$

Then, $\omega_{\text {opt }}(k)$ can be calculated as

$$
\boldsymbol{\omega}_{\mathrm{opt}}(k)=\boldsymbol{R}_{X X}^{-1}(k) \boldsymbol{R}_{X d}(k),
$$

where $\boldsymbol{R}_{X X}(k)=\boldsymbol{X}^{T}(k) \boldsymbol{X}(k)$ and $\boldsymbol{R}_{X d}(k)=\boldsymbol{X}^{T}(k) \boldsymbol{d}(k)$. There is a high computational complexity in the straightforward computation of $\boldsymbol{R}_{X X}{ }^{-1}(k)$, and therefore, the weight vector is updated in a recursive manner according to the matrix inversion lemma [26] as

$$
\begin{aligned}
\boldsymbol{R}_{X X}^{-1}(k) & =\lambda^{-1}\left[\boldsymbol{R}_{X X}^{-1}(k-1)-\gamma^{-1}(k) \boldsymbol{P}(k)\right] \\
\boldsymbol{R}_{X d}(k) & =\lambda \boldsymbol{R}_{X d}(k-1)+d(k) \boldsymbol{x}(k) \\
\boldsymbol{P}(k) & =\boldsymbol{R}_{X X}^{-1}(k-1) \boldsymbol{x}(k) \boldsymbol{x}^{T}(k) \boldsymbol{R}_{X X}^{-1}(k-1), \\
\gamma(k) & =\boldsymbol{x}^{T}(k) \boldsymbol{R}_{X X}^{-1}(k-1) \boldsymbol{x}(k)+\lambda
\end{aligned}
$$

where $k \geq 0$. The initial values are calculated as $\boldsymbol{R}_{X X}{ }^{-1}(-1)$ $=\psi \boldsymbol{I}$ and $\boldsymbol{R}_{X d}(-1)=[0,0, \ldots, 0]^{T}, \boldsymbol{I}$ represents the identity matrix, $\psi$ is a constant and $0<\psi<1$.

The multi-reference adaptive algorithms (also called the multichannel adaptive algorithms) can then be obtained by replacing $\boldsymbol{x}(k)$ and $\boldsymbol{\omega}(k)$ above with $\boldsymbol{X}_{\boldsymbol{J}}(k)=\left[\boldsymbol{x}_{1}{ }^{T}(k)\right.$, $\left.\boldsymbol{x}_{2}{ }^{T}(k), \ldots, \boldsymbol{x}_{J}{ }^{T}(k)\right]^{T}$ and $\boldsymbol{W}_{J}(k)=\left[\omega_{1}{ }^{T}(k), \omega_{2}{ }^{T}(k), \ldots, \omega_{J}{ }^{T}(k)\right]^{T}$, respectively, where $\boldsymbol{x}_{j}^{T}(k)$ and $\boldsymbol{\omega}_{j}^{T}(k)$ represent the input and weight vector of the $j^{\text {th }}$ adaptive filter, respectively, and $j=1,2, \ldots, J$. For the sensing system shown in Fig. $11, J=6$.

The typical power-density spectrum (PDS) of the primary receiving $\mathrm{SCM}$ output and the corresponding output of the ANC based on multichannel LMS and RLS algorithms are shown in Fig. 13(a), (b), and (c), 

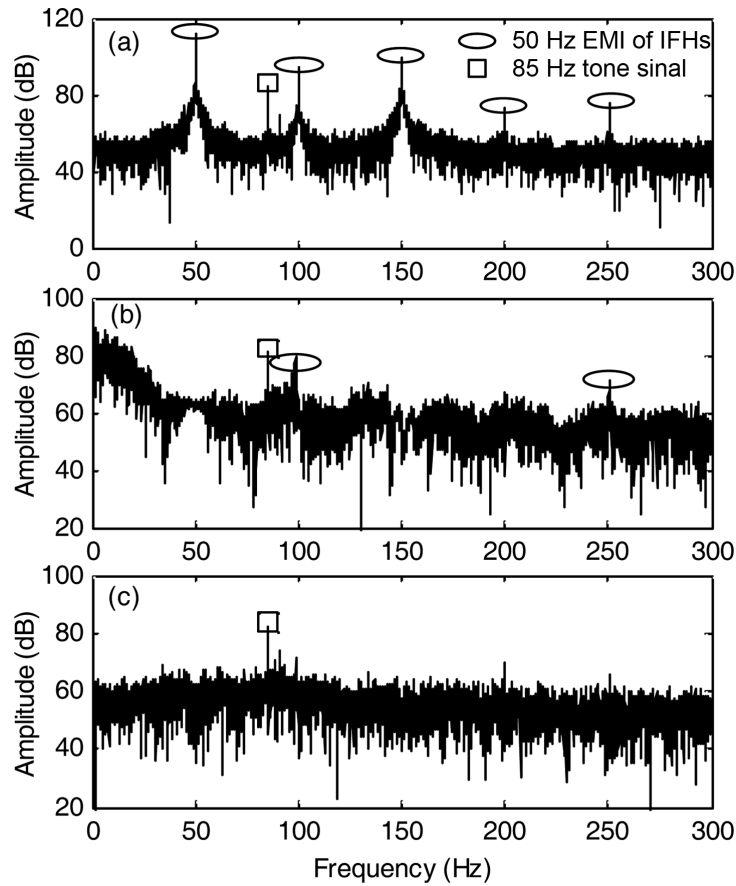

Fig. 13. PDS (a) before EMI cancelling, after EMI cancelling through (b) LMS, and (c) RLS algorithms
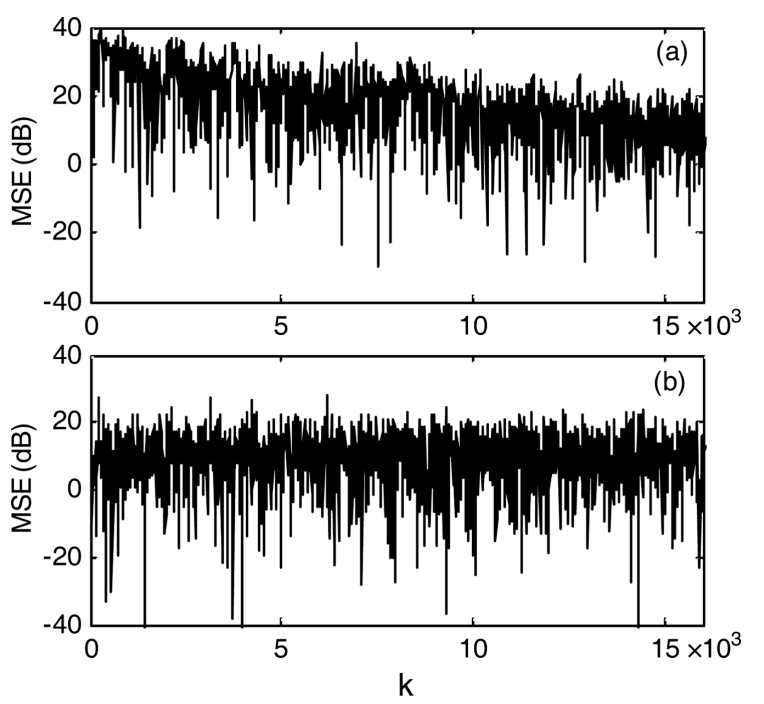

Fig. 14. Learning curves of (a) LMS and (b) RLS algorithms.

respectively. The learning curves of the LMS and RLS algorithms are shown in Fig. 14(a) and (b) respectively, where $\operatorname{MSE}(\mathrm{dB})=10 \log 10\left[e^{2}(k)\right]$. Note that $\mu=2 \times 10^{-5}$, $\lambda=0.99, \psi=10^{-4}$, and $M=32$.

Figure 13(a) shows that the $85-\mathrm{Hz}$ single tone signal is submerged in strong EMI from IFHs with SIR and SINR values of $-27.30 \mathrm{~dB}$ and $-27.31 \mathrm{~dB}$, respectively. Figure 13(b) and (c) show that the SIR gains for the LMS and RLS algorithms are $32.73 \mathrm{~dB}$ and $32.92 \mathrm{~dB}$, respectively,
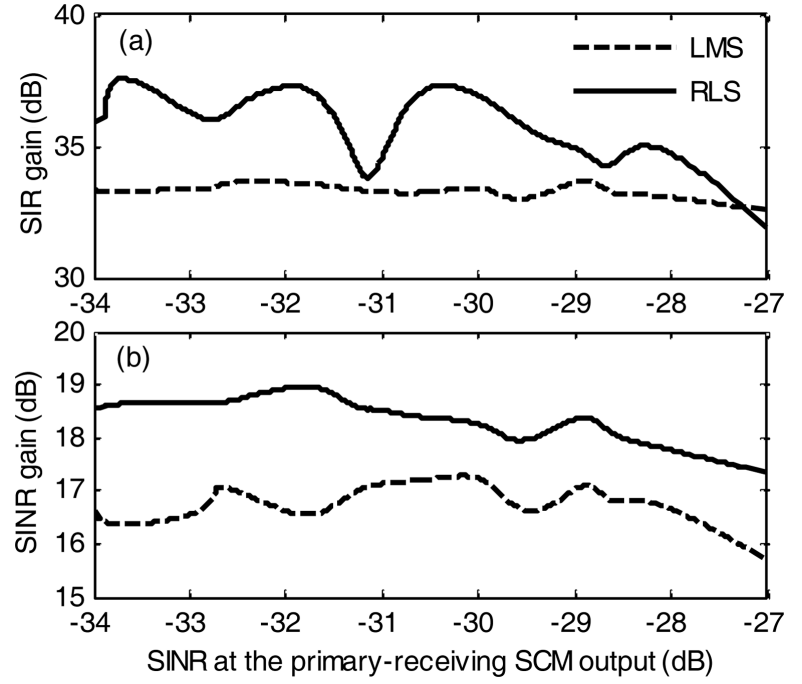

Fig. 15. (a) SIR gains and (b) SINR gains in different signal powers.

and therefore, ANC techniques can effectively reduce the EMI from IFHs. The EMI remaining in (c) is less than in (b), and thus, the RLS algorithm may have a better performance than the LMS. Figure 14 shows that a faster convergence is presented in (b) for the RLS algorithm, in which the cost of increased computational complexity and running time must be paid.

To verify this sensing system further, experiments with different TEC signal powers have been conducted. The strength of the EMI from IFHs was kept constant, and the transmitting powers of the TEC signal varied for different values. For each of the signal powers, five data sets were recorded and processed, and the SIR and SINR gains at the canceller output were estimated and averaged. The configurations of the multichannel adaptive algorithms were the same as above. The final results are shown in Fig. 15.

Figure 15 shows that when the SINR at the primary receiving $\mathrm{SCM}$ output ranges from $-34 \mathrm{~dB}$ to $-27 \mathrm{~dB}$, an average of $36 \mathrm{~dB}$ and maximal $38 \mathrm{~dB}$ of SIR gains based on the multichannel RLS algorithm could be obtained, and the SIR gains for the multichannel LMS algorithm might be slightly less. It also shows that the average SINR gain is less than the average SIR gain because the uncorrelated noise, such as background white noise in reference SCMs, will be superposed at the output of the adaptive filters. In addition, the SIR gains of $36 \mathrm{~dB}$ and $33 \mathrm{~dB}$ could still be obtained for the multichannel RLS and LMS algorithms, respectively, even when the SINR becomes as low as $-34 \mathrm{~dB}$. This shows that the system is especially suitable for a weak TEC signal coupled with strong EMI. Finally, the SIR and SINR gains tend to 
decrease with the TEC signal power increasing since the signal components leaking into the reference inputs tend to increase, and as a result, the signal distortion at the noise canceller output would increase.

\section{Conclusion}

In this paper, we propose a magnetic field sensing system, in which the single primary receiving and multiple reference SCMs are deployed locally and orthogonally. The SCMs are optimized, built, and tested. The magnetic field sensing system is constructed using these SCMs and tested with real EMI from IFHs in laboratory environment. Three fundamental conclusions can be obtained from this study:

1. An average of $36 \mathrm{~dB}$ and $18 \mathrm{~dB}$ of SIR and SINR gains could be obtained, respectively, with six channels of local reference sensors, using the multichannel RLS algorithm.

2. By placing the matching capacitor in parallel with the coil and tuning the resonant frequency to the TEC carrier frequency, the SCM bandwidth can be optimized to be as low as a few Hertz, which is significantly important for TEC signal reception at SLF and lower frequencies. Combined with the optimization of the diameter of the copper wire and the number of turns, the SCM can be designed to achieve high sensitivity and small size further.

3 . This sensing system is effective for interference cancelling at TEC downlink sites, especially in the case of strong local EMI. Thus, reliable downlink signal reception could be achieved using this system.

It is desirable to investigate the beamforming, as well as the stochastic resonance-based algorithms and their applications in signal detection for Through-the-Earth Communication. In addition, the crosstalk and its compensation between the SCMs of the sensing system will be an area for future investigation.

\section{Acknowledgment}

This research was supported by the National Natural Science Foundation of China (41631072). The authors wish to thank Wen-bin LI for his assistance in the experiments, who comes from Wuhan Maritime Communication Research Institute, China.

\section{References}

[1] M. R. Yenchek, G. T. Homce, N. W. Damiano, and J. R. Srednicki, IEEE Trans. Ind. Appl. 48, 1700 (2012).
[2] F. H. Raab, IEEE Trans. Ind. Appl. 24, 212 (1988).

[3] F. H. Raab, IEEE Trans. Comm. 43, 2995 (1995).

[4] D. Madurasinghe and E. O. Tuck, IEEE J. Oceanic Eng. 19, 193 (1994).

[5] Zhang Jiawei, Jiang Runxiang, and Gong Shenguang, Journal of Harbin Engineering University 35, 931 (2014).

[6] Lincan Yan, J. A. Waynert, and C. Sunderman, IEEE Trans. Ind. Appl. 49, 1979 (2013).

[7] V. Bataller, A. Muñoz, P. M. Gaudó, A. Mediano, J. A. Cuchí, and J. L. Villarroel, Radio Sci. 45, RS6015 (2010).

[8] V. Bataller, A. Muñoz, P. Molina, A. Mediano, J. A. Cuchí, and J. L. Villarroel, J. Comm. 4, 284 (2009).

[9] B. Yan, W. H. Zhu, L. S. Liu, K. Liu, and G. Y. Fang, IEEE Sens. J. 15, 1139 (2015).

[10] S. Tumanski, Meas. Sci. Technol. 18, R31 (2007).

[11] H. C. Séran and P. Fergeau, Rev. Sci. Instrum. 76, 0445021 (2005).

[12] A. Grosz and E. Paperno, IEEE Sens. J. 12, 2719 (2012).

[13] B. Yan, W. H. Zhu, L. S. Liu, K. L. and G. Y. Fan, IEEE Trans. Magn. 49, 5294 (2013).

[14] S. Bae, Y. K. Hong1, J. Lee, J. Park, J. Jalli, G. S. Abo1, H. M. Kwon, and C. K. K. Jayasooriya, J. Magn. 18, 43 (2013).

[15] D. A. Chrissan, Statistical Analysis and Modeling of Low-Frequency Radio Noise and Improvement of LowFrequency Communications, Tech. Rep., The Off. of Nav. Res., Washington D. C. (1998).

[16] F. H. Raab, Radio Sci. 45, 1 (2010).

[17] A. C. Fraser-Smith, Low-Frequency Radio Noise, in Handbook of Atmospheric Electrodynamics, CRC Press, Boca Raton (1995) pp. 290-310.

[18] M. Shao and C. L. Nikias, Proc. IEEE 81, 986 (1993).

[19] B. Widrow, J. R. Glover, J. M. McCool, J. Kaunitz, C. S. Williams, R. H. Hearn, J. R. Zeidler, E. Dong, Jr. and R. C. Goodlin, Proc. IEEE 63, 1692 (1975).

[20] A. Rhouni, G. Sou, P. Leroy, and C. Coillot, IEEE Sens. J. 13, 159 (2013).

[21] C. Coillot, J. Moutoussamy, P. Leroy, G. Chanteur, and A. Roux, Sens. Lett. 5, 167 (2007).

[22] J. Vrbancich, Magnetic Field Distribution and Design of Helmholtz Coils, Tech. Rep., Materials Research Laboratory, Maribyrnong (1991).

[23] W. M. Frix, G. G. Karady, and B. A. Venetz, IEEE Trans. Power Delivery 9, 100 (1994).

[24] K. Mohamadabadi and M. Hillion, IEEE Sens. J. 14, 3076 (2014).

[25] Low-Noise JFETs--Superior Performance to Bipolars. Online, Available: http:/www.vishay.com/docs/70599/ 70599.pdf.

[26] Paulo S. R. Diniz, Adaptive Filtering Algorithms and Practical Implementation (Fourth Edition), Springer, New York (2013) pp. 209-213. 